\title{
Verso una terminografia per il traduttore giuridico
}

\author{
Marella Magris \\ SSLMIT di Trieste
}

It is often stated that the structure of a terminological data bank should be developed taking the user's needs into account. Translation-oriented terminology is certainly no exception to this rule. The creator of a data bank for legal terminology should assess the specific problems typically found in legal translation as well as the use of terminological resources by legal translators. However, users' needs and users' behaviour are extensively investigated in specialised lexicography, but seem to be a rather under-researched area in terminology and terminography. By drawing on relevant lexicographic experience, some empirical observations on the terminological needs of LSP translators, and translationoriented terminography with particular reference to legal terminology, this study aims at developing a list of 'questions' (related to terminological problems), to which a legal translator should find answers in a properly constructed and reliable language resource.

\section{Introduzione}

È stato spesso sottolineato che la struttura di una risorsa terminografica deve essere concepita a partire dalle esigenze dell'utente: pertanto, per creare una banca dati $\mathrm{o}$, più in generale, una qualsiasi raccolta terminografica orientata alla traduzione giuridica, sarebbe opportuno valutare innanzitutto quali siano i problemi terminologici tipici di questo genere di traduzione. Al riguardo mancano tuttavia, per quanto è a nostra conoscenza, non solo studi empirici relativi all'ambito specifico qui in oggetto, ma anche, più in generale, indagini su vasta scala sull'uso delle risorse terminografiche da parte di traduttori. Nel presente articolo si cercherà dunque di delineare un quadro delle esigenze del traduttore giuridico avvalendosi di esperienze provenienti da settori affini e adattandole ai problemi specifici della lingua del diritto.

\section{Alcuni spunti dalla ricerca empirica e dalla teoria}

Una prima serie di indicazioni può essere tratta da uno studio empirico condotto da Duvå \& Laursen (1994) sull'uso di dizionari specializzati nel corso del processo traduttivo. L'esperimento riguardava testi e dizionari di economia, ma i risultati possono essere applicati mutatis mutandis anche alla traduzione giuridica. Le ricercatrici hanno chiesto a un gruppo di soggetti (traduttori professionisti e semiprofessionisti) di tradurre un testo economico e di registrare in un protocollo i problemi incontrati, $\mathrm{i}$ 
dizionari consultati per risolverli, l'esito, il tempo impiegato ecc.. Come era prevedibile, la maggior parte dei problemi è risultata riguardare l'identificazione dell'equivalente di un termine: in una delle prove, ad esempio, 264 problemi sono risultati legati all'equivalenza interlinguistica, a fronte di 2 soli problemi concernenti esclusivamente la fase di ricezione del testo di partenza e di 41 problemi relativi alla fase di produzione del testo di arrivo (Duvå \& Laursen 1994: 257). Tuttavia, come viene sottolineato dalle due autrici, il problema di non conoscere l'equivalente coinvolge un certo numero di altri problemi legati a conoscenze linguistiche o specialistiche, problemi che vengono qui ripresi e integrati da esempi relativi alla terminologia giuridica per la coppia di lingue tedesco e italiano:

\section{a) incertezza concernente il contenuto semantico di una parola}

Il soggetto cerca un termine sul dizionario e trova più equivalenti, di cui sceglie quello che gli sembra più plausibile. Un esempio applicato al diritto potrebbe essere dato dal termine einstweilige Verfügung, per il quale sul dizionario Troike-Strambaci \& Helffrich Mariani (1999) si trovano disposizione preliminare, disposizione temporanea, provvedimento d'urgenza. Senza ulteriori indicazioni è difficile che il traduttore scelga da questo elenco il traducente corretto, che è l'ultimo indicato e anche quello più dissimile lessicalmente dal termine tedesco. La semplice elencazione di traducenti è un procedimento attuato con frequenza in quest'opera lessicografica, mentre un altro dizionario giuridico, Conte \& Boss (1995), specifica spesso tra parentesi alcuni sinonimi o quasi sinonimi per distinguere $\mathrm{i}$ diversi significati di una parola polisemica, ad es.: Antrag: (1. Gesuch) istanza, domanda, (2. Angebot) offerta, (3. Vorschlag) proposta $[\ldots]$.

b) incertezza concernente la collocazione della parola nell'universo

In parole più semplici, il soggetto si chiede se possa utilizzare un determinato termine in un preciso contesto. Un esempio applicato al diritto potrebbe riguardare l'uso dei termini Antragssteller e Antragsgegner per un determinato tipo di azione giudiziale. Ma senza andare così nel dettaglio, soltanto di rado i dizionari giuridici indicano il settore del diritto (civile, penale ecc.) cui appartiene il termine.

c) incertezza concernente l'uso della parola

A un traduttore può succedere di chiedersi come un determinato termine vada usato, ma anche se un termine reperito in un dizionario venga effettivamente utilizzato. Ad esempio il sintagma klägerische Partei riportato su Troike-Strambaci \& Helffrich Mariani (1999) non sembra comparire con molta frequenza nei testi specialistici. Sempre nello stesso dizionario, alla voce italiana delibazione si trova come equivalente Delibation, termine in realtà inesistente nel linguaggio giuridico tedesco. Il fatto che Delibation sia seguito da una breve spiegazione del concetto ("Beschluß über die Vollstreckung eines ausländischen Urteils") dovrebbe mettere in guardia il traduttore dall'usare il calco così com'è; tuttavia, ciò non giustifica la scelta delle autrici del dizionario, tanto più che un traducente 
corretto viene fornito alla sottovoce delibazione di sentenza straniera (Anerkennung ausländischer Entscheidung).

d) incertezza concernente l'argomento

Alcuni termini richiedono un approfondimento concettuale superiore a quello descritto ai punti a) e b). Un esempio si trova in Mayer (1998: 4345): come dimostra l'autore, per determinare se tradurre il termine italiano giudice con Richter o Gericht non è sufficiente confrontare le rispettive definizioni né i relativi sistemi concettuali, ma è necessario acquisire conoscenze più approfondite tramite la lettura di articoli specifici della Costituzione e del Grundgesetz e dei codici di procedura civile italiano e tedesco.

e) incertezza concernente le possibilità combinatorie di una parola

La trattazione delle collocazioni è uno degli aspetti più curati in Troike-Strambaci \& Helffrich Mariani (1999). Alla voce Urkunde vengono elencate ad esempio le espressioni eine Urkunde beibringen, beistellen, vorlegen e molte altre. Anche il dizionario Conte \& Boss (1995) riporta numerose collocazioni. Questa attenzione, insolita in dizionari specializzati relativi ad altre discipline, testimonia l'importanza dell'aspetto collocazionale nel linguaggio giuridico.

Dallo studio di Duvå e Laursen (1994) emerge che i dizionari specializzati consultati non hanno dato affatto risposta ai quesiti dei traduttori. Le studiose concludono che i dizionari "tradizionali" non sono adatti alla traduzione e auspicano che la lessicografia specializzata integri sempre più principi della terminografia. In particolare, un dizionario specializzato "ideale" dovrebbe essere basato su un ordine non alfabetico bensì sistematico e contenere informazioni enciclopediche sull'argomento, definizioni, etichette che specifichino il campo tematico cui appartiene il termine, esempi e collocazioni. Senza voler entrare nella polemica relativa alla distinzione tra lessicografia specializzata e terminografia, vale la pena ricordare che la branca della terminografia che qui più interessa, ossia quella orientata alla traduzione, è già caratterizzata da un approccio misto, che integra principi lessicografici e terminografici al fine di rispondere alla complessa tipologia dei problemi (linguistici, concettuali e pragmatici) che possono sorgere durante il processo traduttivo.

Una lista di problemi terminologici che possono presentarsi nella traduzione specializzata è stata stilata da Cabré (1999: 216):

- problemi riguardanti la lingua B (ovvero la lingua di partenza):

- conoscere o precisare il significato di un'unità terminologica del testo di partenza

- confermarne il carattere specialistico

- conoscere le denominazioni alternative e le relative condizioni d'uso nei testi, ecc.

- problemi riguardanti la lingua A (ovvero la lingua di arrivo):

- sapere se esiste un'unità terminologica equivalente nella lingua di arrivo 
- qualora non esista, sapere a quali strumenti ricorrere per adattare o creare una denominazione adeguata

- conoscere la denominazione più adeguata tenendo conto del tema, della prospettiva del lavoro e del destinatario

- sapere se un termine debba essere usato dal punto di vista grammaticale in modo speciale o restrittivo

- conoscere le caratteristiche combinatorie dei termini

- conoscere la fraseologia dell' ambito specialistico

- conoscere le denominazioni alternative per uno stesso concetto e le relative condizioni pragmatiche

- assicurarsi che la denominazione selezionata corrisponda esattamente al concetto da designare, ecc.

Come si vedrà, molti dei punti elencati si ritrovano anche nella lista, da noi stilata, di quesiti cui dovrebbe dare risposta una risorsa terminografica (cfr. sez. 3): in altri casi, invece, si tratta di problemi che possono essere risolti soltanto con una conoscenza approfondita della lingua speciale oggetto della traduzione, nonché di principi della teoria della terminologia e di analisi del testo (cfr. sez. 4).

A conclusione di questa sezione, si ritiene opportuno accennare ad alcuni esperimenti condotti presso la SSLMIT di Trieste sull'uso di una banca data terminologica da parte di traduttori (Musacchio \& Magris 2001; Magris \& Musacchio 2001). Lo scopo principale non era evidenziare i problemi tipici incontrati, bensì verificare l'efficacia della banca dati rispetto ad altri strumenti di consultazione. Va detto inoltre che gli esperimenti sono stati condotti su un numero troppo limitato di partecipanti per poterne trarre dati statistici. Pur tuttavia, poiché le prove sono state effettuate su testi di argomento giuridico, si possono fare alcune brevi considerazioni. Uno dei problemi incontrati più di frequente dai soggetti riguardava la corretta identificazione di alcuni termini, formalmente identici a parole della lingua standard; altri aspetti per i quali la risorsa terminografica si è dimostrata più efficace rispetto agli altri strumenti a disposizione sono ad esempio la possibilità di effettuare un approfondimento tematico ragionevolmente rapido per risolvere alcuni problemi di comprensione, la corretta identificazione di sinonimi e iperonimi, l'acquisizione di alcune conoscenze di natura enciclopedica.

\section{Le domande del traduttore giuridico}

Partendo da quanto esposto nella sezione precedente, si presenterà ora una lista (da non considerarsi esaustiva) di potenziali quesiti cui la terminografia giuridica dovrebbe dare risposta.

I quesiti vengono suddivisi per chiarezza in una serie riguardante l'analisi del testo di partenza e in una concernente la produzione del testo di arrivo. 


\section{Analisi del testo di partenza}

\section{La parola o il sintagma $X$ è un termine giuridico?}

Il problema di identificare come tale un termine è abbastanza frequente nella lingua giuridica, dati i rapporti particolari che questa ha con il lessico della lingua comune. Molti termini giuridici sono infatti parole mutuate dalla lingua standard e sottoposte poi a un processo di rideterminazione semantica. Nella terminologia della procedura civile, per esempio, Urkunde non va inteso nel suo significato generico, bensì nell'accezione più specifica di "Gedankenäußerung, die durch Schriftzeichen verkörpert wird” (Musielak 1995: 52). Nell'identificare le unità terminologiche, inoltre, il traduttore farà bene a tener presente che, più di quanto accada in altri settori, nel diritto anche i verbi acquisiscono spesso uno "statuto terminologico" (Krieger 2002: 235).

- X è un termine specifico dell'ambito tematico di cui tratta il testo, o attiene al diritto in generale? Per rimanere all'esempio di Urkunde, il termine così come è stato definito prima riguarda soltanto la procedura civile, mentre nel diritto penale ha un significato più esteso.

- X è un termine a sé stante, oppure è una variante di un termine Y? La corretta identificazione di una variante può evitare di incorrere in errori di comprensione: particolarmente importante è evitare di scambiare la forma abbreviata di un termine per un iperonimo dello stesso (cfr. Hohnhold 1990: 51). Un esempio è dato dal termine sospensione del processo che, in un atto giurispruden-ziale, è sicuramente la variante abbreviata di un sintagma più esteso. Il traduttore dovrà capire se si tratta di una sospensione necessaria (da tradurre con Aussetzung des Verfahrens) o di una sospensione su istanza delle parti (in questo caso il traducente corretto sarà Ruhen des Verfahrens). Similmente, nel diritto delle obbligazioni concreditore può comparire come forma abbreviata sia di concreditore in solido che di concreditore parziario; sarà spesso solo il contesto a chiarire se si tratta di solidarietà o parziarietà (Nicolosi 2003-04).

\section{Quale concetto designa il termine X?}

Una prima analisi del concetto designato dal termine, solitamente tramite la lettura della relativa definizione, può servire al traduttore per acquisire una parte di quelle conoscenze specialistiche indispensabili a portare a termine l'incarico di traduzione, nonché come primo elemento per valutare l'equivalenza concettuale con un potenziale traducente. Va rilevato, tuttavia, che per le specifiche esigenze del diritto non sempre i termini giuridici sono definiti in modo rigoroso e che la terminologia non può sostituirsi al diritto stesso e ricercare precisione dove invece vi è vaghezza (cfr. Krieger 2002: 241). Si pensi ad esempio a quei termini, come buona fede, buon costume, ragionevolezza, diligenza, designanti quelle che nel diritto vengono chiamate "clausole generali". Una loro definizione concettuale è impossibile "senza ricorrere al riferimento esterno dato 
dai parametri di giudizio della morale sociale che variano nel tempo" (Rossi: 17).

- Si tratta di termine qualificatore o fattuale? Il primo tipo di termine trae la propria qualificazione da una norma, mentre il secondo assume un particolare valore semantico attraverso l'uso che ne fanno i giuristi (Snel Trampus 1989: 69). La differenza si ripercuote sia sul tipo di definizione che si può individuare (definizione legale o meno), sia sulla monoreferenzialità (v. oltre). La distinzione ha inoltre rilevanza anche nella dimensione interlinguistica, in quanto non sempre per un termine qualificatore nella lingua di partenza si potrà reperire un equivalente che sia anch'esso qualificatore nella lingua di arrivo. Ad esempio, il tipo di obbligazione denominato Schickschuld è disciplinato nel codice civile tedesco ma non in quello italiano. Il concetto tuttavia è noto anche in Italia, essendo ammesso nella prassi degli scambi economici, ed è designato dal termine obbligazione con prestazione da avviarsi a destinazione (Nicolosi 2003-04).

- Qual è la posizione del concetto all'interno del sistema concettuale? Assieme alla definizione, l'individuazione degli eventuali iperonimi, iponimi, coiponimi ecc. faciliterà al traduttore il confronto con l'equivalente nella lingua di arrivo. Tuttavia, come già rilevato per le definizioni, la costruzione di sistemi concettuali in ambito giuridico non si presenta sempre agevole. Spesso, infatti, il terminologo che esamini le classificazioni proposte dalla dottrina si troverà di fronte a sistemi diversi: ogni singolo giurista tende a costruire una classificazione propria dell'ordinamento nel quale si trova a operare, in quanto, a prescindere da alcuni assiomi fondamentali, le tassonomie giuridiche non sono necessariamente così particolareggiate da impedire costruzioni alternative (Rossi: 22).

- Vi sono altri elementi che limitano o ampliano l'estensione del concetto? Alcune informazioni di natura enciclopedica possono influenzare l'applicazione di un concetto a determinati casi concreti. Con il termine periodo di riposo, ad esempio, si definiscono le due interruzioni giornaliere concesse alla lavoratrice madre durante il primo anno di vita del bambino (il cosiddetto "permesso per allattamento"). Nella scheda terminografica relativa è opportuno inserire l'indicazione che in Italia esso può essere riconosciuto anche al padre lavoratore, in alternativa alla madre lavoratrice dipendente che non se ne avvalga (Reggio 19992000). Il termine, pertanto, potrà essere usato anche in riferimento a soggetti maschili (diversamente dal suo equivalente tedesco Stillzeit).

- Il termine è monoreferenziale?

Come in molte altre lingue speciali, anche nel diritto la monoreferenzialità postulata da alcuni linguisti si rivela sovente un'illusione. All'interno del diritto delle obbligazioni, ad esempio, il termine stesso obbligazione ha tre significati distinti: 1) il rapporto giuridico tra due persone, di cui una è debitrice dell'altra; 2) la posizione passiva di tale rapporto; 3) un particolare tipo di titolo di credito (Nicolosi 2003-04). 
- L'interpretazione del termine è ancora relativamente aperta? Questo problema riguarda soprattutto i termini fattuali, il cui significato non è stato (ancora) stabilito rigorosamente da una norma. Un esempio è dato dal termine famiglia di fatto, cui nella dottrina vengono dati due significati diversi: l'unione di due persone non legate da loro da vincoli matrimoniali (ed eventualmente dei figli da esse procreati) oppure il legame esistente tra due persone legate da matrimonio esclusivamente religioso (in particolare da un matrimonio canonico non registrato) (Lampis 1996-97).

- Il termine designa concetti diversi a seconda dell'ambito geografico in cui viene usato? Questo secondo aspetto può riguardare anche i termini qualificatori, qualora una stessa lingua venga usata in più ordinamenti giuridici nazionali o internazionali. Il problema si pone in modo evidente per il tedesco, utilizzato nella RFT, in Austria, Svizzera, Alto Adige e nel Liechtenstein. Inoltre, sia per il tedesco che per l'italiano bisogna considerare anche il livello comunitario, che darà origine a una propria varietà di terminologia giuridica. Nel settore della tutela dei dati personali, per esempio, il termine responsabile designa due concetti distinti a livello nazionale e comunitario. Per l'ordinamento italiano, il responsabile è la persona fisica, la persona giuridica, la pubblica amministrazione o qualsiasi altro ente, associazione od organismo preposti dal titolare al trattamento di dati personali; per la direttiva CEE, invece, responsabile del trattamento è figura coincidente proprio con il titolare (Ciriello 1998-99).

\section{Il termine presenta particolari connotazioni?}

Oltre al significato referenziale, il termine può veicolare altre componenti di significato, ad esempio segnalando l'atteggiamento di un giurista nei confronti di un determinato tema. Chi usi il termine famiglia irregolare al posto di famiglia di fatto o fecondazione artificiale anziché fecondazione assistita esprime in modo più o meno esplicito il proprio dissenso.

\section{I termini $\mathrm{X}$ e $\mathrm{Y}$ sono sinonimi?}

Si tratta di verificare se due termini che compaiono all'interno di uno stesso testo siano sinonimici, al fine di garantire una corretta comprensione del testo stesso.

- Sono sinonimi pieni? Al fine di accertare il grado di sinonimia si applicherà sul piano intralinguistico il medesimo procedimento di confronto delle definizioni e delle relazioni concettuali utile a livello interlinguistico per determinare il grado di equivalenza.

- Se sono sinonimi parziali, quali sono le differenze? I termini tedeschi Kündigungsschutz e Kündigungsverbot, per esempio, pur designando lo stesso concetto (l'impossibilità di licenziare il lavoratore in determinate situazioni), esprimono una prospettiva diversa (il primo quella del lavoratore, il secondo quella del datore di lavoro) (Reggio 19992000). Si tratterà dunque di pseudosinonimi, che non risulteranno intercambiabili in qualsiasi contesto. Un altro esempio è dato dai termini prova e mezzo di prova: pur essendo usati spesso come sinonimi, il 
primo indica più propriamente il giudizio, il risultato ottenuto con il secondo.

\section{La collocazione in cui compare il termine $X$ ha un significato specia- listico?}

Nella fase di analisi del testo di partenza il problema posto dalle collocazioni non sarà tanto quello di determinare, per esempio, con quale verbo si collochi un determinato sostantivo, ma piuttosto di sapere se una particolare collocazione abbia un significato definito con precisione. Come sottolinea Hohnhold (1990: 34), infatti, anche le collocazioni possono essere oggetto di una definizione, anche se di tipo diverso rispetto a quelle dei termini, perché diverso è il rapporto di designazione (i termini designano concetti, le collocazioni invece rappresentano situazioni, fattispecie). Le collocazioni potranno dare origine a tutti i problemi già elencati per i termini. Trovandosi di fronte le due espressioni esibire un documento e produrre un documento, un traduttore potrebbe ad esempio supporre che tra esse vi sia un rapporto di sinonimia piena: in realtà, mentre la prima indica un'azione richiesta dal giudice, la seconda esprime un atto spontaneo di una delle parti di un processo. È pertanto importante che una raccolta terminografica tratti anche questo tipo di designazioni più complesse, anche se ciò richiede la soluzione di alcuni problemi tecnici e l'adozione di scelte ben ponderate. Difficoltà tecniche sorgono ad esempio per la registrazione delle formule fisse, in cui non sempre è possibile individuare una parola chiave che funga da lemma.

\section{Produzione del testo di arrivo}

\section{Il termine $\mathrm{Z}$ viene usato in testi specialistici o soltanto in opere ter- minografiche/lessicografiche?}

Il traduttore che reperisca un determinato traducente in un dizionario è impossibilitato a verificarne la validità, poiché nelle opere lessicografiche non viene indicata la fonte da cui sono tratti i termini e gli altri dati forniti. Chi consulta una raccolta terminografica può invece verificare se il termine è stato tratto da un testo specialistico o da materiale lessicografico, o ancora se si tratta di una proposta del terminografo per colmare una lacuna nella lingua di arrivo.

- Se viene usato in testi specialistici, in quali tipi di testi? Nella traduzione giuridica acquista rilevanza soprattutto la ripartizione in linguaggio legislativo, giurisprudenziale e dottrinale. I termini soggetto attivo e soggetto attivo della domanda giudiziale, ad esempio, sono sinonimi di attore utilizzati esclusivamente in testi dottrinali (in particolare in manuali giuridici), che risulterebbero del tutto inadeguati per tradurre il termine Kläger all'interno di una sentenza.

- In quale sottosettore del diritto o in quale ambito tematico? I due termini tedeschi Aussetzung des Verfahrens e Ruhen des Verfahrens, già citati, vengono usati relativamente al processo di cognizione (Erkenntnisverfahren), mentre per il processo di esecuzione (Zwangs- 
vollstreckung) si parlerà di (einstweilige) Einstellung (Musielak 1998: 170, 337).

- In testi di quale area geografica? Molti termini potranno essere tradotti correttamente soltanto qualora si conosca la "destinazione geografica" della traduzione (e il relativo ordinamento giuridico). Ristoro agrituristico andrà ad esempio tradotto con Straußwirtschaft (termine contenuto nella Gaststättengesetz) se il testo è destinato alla RFT, con Buschenschank se si tratta dell'Austria o dell'Alto Adige (Benetti 199899).

- In testi in lingua originale o tradotti? Nella traduzione giuridica può essere spesso molto utile poter rintracciare "precedenti" terminologici, ossia traducenti già proposti da altri autori o traduttori per termini che esprimano determinate peculiarità della cultura di partenza. In una raccolta terminografica sarebbe comunque opportuno segnalare esplicitamente l'origine di tali espressioni, in quanto vi potrebbero essere differenze più o meno sostanziali rispetto alla terminologia originale. Nel campo del diritto sindacale, Cosmai (1993-94) ha analizzato i traducenti impiegati nella traduzione italiana di una monografia tedesca, individuando tra l'altro il ricorso frequente a calchi. I sinonimi Arbeit nach Vorschrift e Dienst nach Vorschrift, ad esempio, vengono resi con servizio secondo le disposizioni e sciopero zelante, mentre nella manualistica italiana si utilizzano termini come ostruzionismo e sciopero pignolo, che sono legati a quello tedesco per lo meno da un'equivalenza parziale.

Quale concetto designa il termine $Z$ ?

- Si tratta di termine qualificatore o fattuale?

- Qual è la sua posizione all'interno del sistema concettuale?

- Vi sono altri elementi che ne limitano l'estensione?

- Il termine è monoreferenziale?

- L'interpretazione del termine è ancora relativamente aperta?

- Il termine designa concetti più o meno diversi a seconda dell'ambito geografico in cui viene usato?

Una volta individuato un potenziale traducente per il termine $\mathrm{X}$ della lingua di partenza, è necessario ripercorrere le varie fasi al fine di determinare in modo definitivo l'equivalenza interlinguistica. Una scheda terminografica dovrebbe "risparmiare" al traduttore tale processo, presentandogli tuttavia in maniera sintetica ma chiara le differenze eventualmente esistenti. Un traduttore che ricercasse il traducente del termine Gutachten (eines Sachverständigen), ad esempio, troverebbe probabilmente molto utili le seguenti informazioni: in italiano, il concetto corrispondente viene designato da due termini diversi (perizia nel diritto processuale penale, consulenza tecnica in quello civile); per quanto attiene il secondo ambito, quello civile, in Germania è forse più frequente il riferimento al soggetto, ovvero al Sachverständiger; sempre in ambito civile, infine, non vi è corrispondenza piena tra i sistemi concettuali italiano e tedesco, in quanto in Germania il Gutachten rientra nei mezzi di prova, 
in Italia no. Il carattere molto "precario" dell'equivalenza interlinguistica nel diritto è stato evidenziato sia da Sandrini (1996) sia da Mayer (1998), i quali hanno proposto due modelli diversi di struttura terminografica che tengono conto della complessità del settore. Il modello di Sandrini (1996: 225), in particolare, rinuncia del tutto a schede bi- o plurilingui: in considerazione della diversità degli ordinamenti giuridici e della conseguente mancanza di equivalenza assoluta, ogni singolo concetto viene documentato separatamente, in schede monolingui. Il collegamento tra le schede nelle diverse lingue viene realizzato poi in modo più o meno diretto a seconda del grado di corrispondenza esistente tra i concetti (Sandrini 1996: 242-251).

\section{Il termine presenta particolari connotazioni?}

Come nella fase di analisi del testo di partenza, anche in questo caso è importante verificare se il termine, oltre alla componente denotativa, abbia altri significati e, in caso positivo, accertare se questi corrispondano alle connotazioni del termine originale.

I termini A, B, C, indicati come sinonimi, sono sinonimi pieni?

Se non lo sono, quali sono le differenze? Si tratta di differenze che riguardano il significato referenziale, di connotazioni diverse o piuttosto dell'espressione di una varietà diatopica, diacronica ecc.? Anche in questo caso, la scheda terminografica dovrebbe segnalare le peculiarità, preferibilmente in forma sintetica (attraverso appositi attributi).

\section{Il termine ha delle varianti?}

\section{Quali sono le collocazioni possibili del termine?}

In fase di produzione del testo di arrivo è importante conoscere quali sono le espressioni effettivamente usate, in quanto una semplice traduzione non porta necessariamente a risultati soddisfacenti. Un equivalente corretto di einen Schlüssel erraten può essere ad esempio ricostruire una chiave, e non indovinare o simili (Trevisan 1998-99).

\section{- Le collocazioni hanno un significato specialistico?}

Vi sono delle particolarità grammaticali? Un termine potrebbe avere nella lingua speciale un genere o plurale diverso rispetto alla parola omonima usata nella lingua comune, reggere una preposizione particolare ecc.. L'importanza di evidenziare questi aspetti viene sottolineata ad esempio da Maidahl Christiansen et al. (1994: 273): mentre il lessicografo che si occupa della lingua comune può fare affidamento sulla competenza dell'utilizzatore del dizionario nella sua lingua materna, il terminografo che analizza una lingua speciale non può fare altrettanto, dato che questa sarà sempre in una certa misura "straniera" dal punto di vista terminologico/concettuale.

Di un termine viene usata anche la forma verbale? Ad esempio il sintagma nominale Aussetzung des Verfahrens può essere trasformato in das Verfahren aussetzen, mentre per il termine Ruhen des Verfahrens è necessario ricorrere a una collocazione, come das Ruhen des Verfahrens anordnen o die Anordnung des Ruhens des Verfahrens aussprechen.

- Dal termine derivano uno o più aggettivi? Dal sostantivo attore pos- 
sono derivare gli aggettivi attorio e attoreo. Mentre alcuni dizionari generali della lingua italiana indicano attorio come forma principale, riportando attoreo unicamente come variante, nel linguaggio giurisprudenziale è sicuramente questa seconda forma a essere utilizzata con maggior frequenza.

\section{Limiti e ulteriori potenzialità della terminografia}

Come si può ricavare dalla lista della sezione precedente (peraltro, come si diceva, non esaustiva), sono molti i punti in cui la terminografia orientata alla traduzione, di approccio descrittivo e testuale, può venire in aiuto al traduttore. Va detto tuttavia che anche questa forma di terminografia andrà comunque integrata da una conoscenza delle caratteristiche fondamentali della lingua speciale e da specifiche competenze di analisi testuale. Rimangono infatti alcuni aspetti terminologici che possono difficilmente trovare risposta in una risorsa terminografica. Per quanto riguarda il livello concettuale, ad esempio, sempre più autori richiamano l'attenzione sulle differenze tra i termini definiti a livello di langue e il loro uso nella parole (cfr. per esempio Gerzymisch-Arbogast 1996 \& Antia 2002). Dal punto di vista stilistico, difficilmente una raccolta terminografica potrà fornire chiarimenti sull'attitudine o meno di una determinata lingua speciale alla variazione sinonimica. Pertanto un traduttore che trovi in una scheda un termine principale seguito da diversi sinonimi non saprà se essi possano essere alternati all'interno di uno stesso testo o se rappresentino piuttosto le preferenze individuali di autori diversi. In modo analogo, solo attraverso la conoscenza della relativa lingua speciale il traduttore saprà se e in quale grado potrà servirsi di varianti di un termine come mezzo coesivo. E solo attraverso la conoscenza delle forme adottate più di frequente in una lingua speciale per la formazione dei termini il traduttore potrà giungere a una proposta adeguata in caso di lacuna terminologica.

Vi è tuttavia un ulteriore contributo che la terminografia può dare, anche se in modo difficilmente quantificabile: può aiutare a risolvere un problema di fondo, ovvero l'insicurezza del traduttore. Nello studio di Maidahl Christiansen et al. (1994: 279) sull'uso dei dizionari specializzati, i traduttori indicavano come problema principale la scarsa conoscenza dell'argomento e della relativa struttura concettuale. Tale insicurezza si è evidenziata anche nel corso degli esperimenti condotti presso la SSLMIT: alcuni traduttori hanno ripetuto più volte frasi quali "Non sono sicuro", "Non ho familiarità con l'argomento", "Non ho mai tradotto testi di questo settore" ecc.. Di solito, il traduttore acquista piano piano familiarità e, con essa, sicurezza, grazie alla consultazione di testi paralleli, lavoro insostituibile per certi versi, ma che richiede molto tempo. Dato che anche il lavoro del terminografo parte dall'analisi, ben più approfondita, di un corpus di testi, il suo obiettivo dovrebbe essere quello di trasmette- 
re il più possibile agli altri le conoscenze così acquisite. Affinché questo trasferimento di saperi sia il più efficace possibile, bisogna operare su due piani.

Il primo riguarda la singola scheda terminografica. Le voci non devono servire soltanto a reperire equivalenti, ma essere considerate piuttosto una chiave d'accesso a una varietà di informazioni di tipo linguistico, concettuale, enciclopedico, pragmatico. Oltre alla definizione, pertanto, risultano preziosi anche campi quali il contesto, le note linguistiche ed enciclopediche, le specificazioni corrispondenti alla suddivisione tematica ecc..

Il secondo piano, importantissimo, è il modo in cui le schede vengono collegate le une alle altre attraverso i rimandi. Quanto più il traduttore avrà modo di "navigare" in modo sistematico da un concetto all'altro, tanto più potrà crearsi un'idea della struttura delle conoscenze dell' ambito tematico in cui sta lavorando.

Attraverso tale combinazione di aspetti micro- e macrostrutturali, la terminografia può davvero aiutare il traduttore giuridico a risolvere almeno una parte dei tanti problemi traduttivi posti da questo complesso e affascinante settore di attività.

\section{Bibliografia}

Antia, Bassey E. (2002). "Il termine: contesto definitorio e contesto d'uso". M. Magris, M. T. Mussachio, L. Rega \& F. Scarpo (a cura di) (2002). Manuale di terminologia. Aspetti teorici, metodologici e applicativi. Milano: Hoepli, 99-114.

Benetti, Federica (1998-99). Indagine terminologica nel settore del turismo con particolare riferimento al contratto di viaggio: tedesco-italiano, italianotedesco. Tesi di laurea non pubblicata, Università degli Studi di Trieste.

Cabré, Maria Teresa (1999). La terminología: representación y comunicación. Elementos para una teoría de base comunicativa y otros artículos. Barcelona: Institut Universitari de lingüística aplicada, Universitat Pompeu Fabra.

Ciriello, Antonella (1998-99). Tutela dei dati personali in Italia, Paesi Bassi e Regno Unito: un'indagine non pubblicata, Università degli Studi di Trieste.

Conte, Giuseppe \& Hans Boss (1995). Dizionario giuridico ed economico, italiano-tedesco; tedesco-italiano. 5. edizione. Milano: Giuffré/München: Beck.

Cosmai, Domenico (1993-94). Il diritto sindacale italiano e tedesco: un'analisi terminologica. Tesi di laurea non pubblicata, Università degli Studi di Trieste.

Duvå, Grete \& Anna-Lise Laursen (1994). "Translation and LSP Lexicography: A User Survey". B. Schaeder \& H. Bergenholtz (a cura di) (1994). Fachlexikographie: Fachwissen und seine Repräsentation in Wörterbüchern. Tübingen: Narr, 247-268.

Gerzymisch-Arbogast, Heidrun (1996). Termini im Kontext: Verfahren zur 
Erschliessung und Übersetzung der textspezifischen Bedeutung von fachlichen Ausdrücken. Tübingen: Narr.

Hohnhold, Ingo (1990). Übersetzungsorientierte Terminologiearbeit. Eine Grundlegung für Praktiker. Stuttgart: InTra.

Krieger, Maria da Graça (2002). "Terminographie juridique et spécificités textuelles". Meta XLVII (2), 233-243.

Lampis, Serena (1996-97). La famiglia di fatto. Cenni sulla fattispecie giuridica e terminologia relativa in Italia e in Germania. Tesi di laurea non pubblicata, Università degli Studi di Trieste.

Magris, Marella \& Maria Teresa Musacchio (2001). "La ricerca terminologica nella traduzione specializzata: un esperimento con approcci diversi". F. Mayer (a cura di) (2001). Language for Special Purposes: Perspectives for the New Millennium. Tübingen: Narr, Vol. 1, 311-319.

Maidahl Christiansen, Lisbeth, Grete Duvå, \& Anna-Lise Laursen (1994). "Das Translationswörterbuch für Fachsprachen: Ein integriertes Konzept”. B. Schaeder \& H. Bergenholtz (a cura di) (1994). Fachlexikographie: Fachwissen und seine Repräsentation in Wörterbüchern. Tübingen: Narr, 269-284.

Mayer, Felix (1998). Eintragsmodelle für terminologische Datenbanken: ein Beitrag zur übersetzungsorientierten Terminographie. Tübingen: Narr.

Musacchio, Maria Teresa \& Marella Magris (2001). "The Contribution of Terminology to Text Analysis in Specialised Translation". El traductor profesional ante el próximo milenio. Proceedings of the Second Conference on Training and Career Development in Translating and Interpreting (Madrid, 17-20/2/1999). Madrid: Universidad Europea-CEES.

Musielak, Hans-Joachim (1998). Grundkurs ZPO. 4., neubearbeitete Auflage. München: Beck.

Nicolosi, Michaela (2003-04). Il diritto delle obbligazioni: cenni sulla fattispecie giuridica e relativa terminologia in Italia e in Germania. Tesi di laurea non pubblicata, Università degli Studi di Trieste.

Reggio, Silvia (1999-2000). La tutela della donna nel diritto del lavoro: un'indagine terminologica in Germania e in Italia. Tesi di laurea non pubblicata, Università degli Studi di Trieste.

Rossi, Piercarlo: "Ontologie applicate e comparazione giuridica: alcune premesse".

http://www.dsg.unito.it/ut/data/papers/ontologie\%20applicate\%20e\%20com parazione\%20giuridica.pdf (consultato il 29/07/2004).

Sandrini, Peter (1996). Terminologiearbeit im Recht: Deskriptiver begriffsorientierter Ansatz vom Standpunkt des Übersetzers. Vienna: Internat. Network for Terminology.

Snel Trampus, Rita D. (1989). La traduzione e i linguaggi giuridici olandese e italiano. Aspetti e problemi. Trieste: Edizioni Italo Svevo.

Trevisan, Silvia (1998-99). La sicurezza informatica: crittologia, firma digitale e protocolli di sicurezza in Internet. Un'indagine terminologica in tre lingue: italiano, francese e tedesco. Tesi di laurea non pubblicata, Università degli Studi di Trieste.

Troike Strambaci, Hannelore \& Elisabeth Helffrich Mariani (1999). Vocabolario italiano tedesco del diritto e dell'economia. 2. edizione. Milano: Giuffrè. 
